\title{
Métodos de determinação da disponibilidade de cobre em substratos
}

\author{
Alceu L Padua Junior²; Cleide Aparecida de Abreu ${ }^{1}$; Pedro Roberto Furlani ${ }^{1}$; Mônica F de Abreu ${ }^{1}$ \\ ${ }^{1}$ Instituto Agronômico, Centro P\&D de Solos e Recursos Ambientais, C. Postal: 28, 13012-970 Campinas-SP; ${ }^{2}$ Pós-graduando da PG- \\ IAC; alceulinares@iac.sp.gov.br
}

\begin{abstract}
RESUMO
Para melhorar a qualidade das plantas produzidas em ambientes protegidos ou semi-protegidos é de fundamental importância a adoção de materiais inertes ou pouco alterados que exerçam a função de solo. Esses materiais são chamados de substratos e apresentam as mais variadas origens e composições, podendo ser orgânicos, minerais ou sintéticos. Dentre os problemas nutricionais, a falta ou o excesso de cobre $(\mathrm{Cu})$ é um dos que mais têm afetado a produção de mudas. Portanto, o objetivo desta pesquisa foi de avaliar métodos que sejam eficientes em determinar o $\mathrm{Cu}$ disponível em substrato. $\mathrm{O}$ experimento foi conduzido em casa de vegetação, usando os substratos à base de fibra de coco, casca de pinus e turfa e a rúcula (Eruca sativa L.) como planta teste. Todos os substratos receberam cinco doses de cobre $\left(0 ; 0,5 ; 1,0 ; 2,0\right.$ e 3,0 mg de $\mathrm{Cu}$ por $\mathrm{dm}^{3} \mathrm{de}$ substrato). Após a colheita da rúcula retirou-se uma amostra de substrato para extração de $\mathrm{Cu}$ usando os seguintes métodos: (DTPA $\mathrm{pH}$ a 2,6), (DTPA + trietanolamina a $\mathrm{pH} 7,3), \mathrm{HNO}_{3}+\mathrm{H}_{2} \mathrm{O}_{2}$ a $30 \%$, $\mathrm{K}_{4} \mathrm{P}_{2} \mathrm{O}_{7}$ e ( $\mathrm{NaOCl}$ a pH 8,5 + DTPA). As soluções ácidas apresentaram maior capacidade extrativa de $\mathrm{Cu}$ para todos os substratos testados. $\mathrm{Na}$ análise individual por substrato, somente a fibra de coco apresentou valores de $\mathrm{R}^{2}$ significativos para todos os métodos. $\mathrm{Na}$ análise conjunta dos substratos, todos os métodos apresentaram coeficientes de determinação significativos $\left(\mathrm{R}^{2}>0,51\right)$, mostrando que esses métodos poderão ser utilizados na avaliação da disponibilidade de $\mathrm{Cu}$ em substratos.
\end{abstract}

Palavras chave: extratores, análise química, micronutriente, fibra de coco, casca de pinus e turfa.

\begin{abstract}
Copper availability determination in substrates

To improve the quality of plants grown under protected or semiprotected environments it is very important to adopt inert or less altered materials to use as soilless media. These materials, called substrates, depending on its origin could be organic, mineral, or synthetic. Among the nutritional problems, the lack or excess of copper $(\mathrm{Cu})$ has been the main factor affecting seedling production. The objective of this research was to evaluate efficient methods to quantify available $\mathrm{Cu}$ in substrates. The experiment was conducted under semi-controlled greenhouse conditions with rucula (Eruca sativa L.) as plant-test grown on three substrates (coir, pinus bark, and peat). All the substrates received five $\mathrm{Cu}$ doses $(0 ; 0.5 ; 1.0 ; 2.0$, and $\left.3.0 \mathrm{mg} \mathrm{dm}^{-3}\right)$. After the plant harvest a substrate sample was collected to extract $\mathrm{Cu}$ by the following methods: DTPA at $\mathrm{pH} 2.6$, DTPA + triethanolamine at $\mathrm{pH} 7.3, \mathrm{HNO}_{3}+\mathrm{H}_{2} \mathrm{O}_{2}$ at $30 \%, \mathrm{~K}_{4} \mathrm{P}_{2} \mathrm{O}_{7}$, and $\mathrm{NaOCl}$ at $\mathrm{pH} 8.5+\mathrm{DTPA}$. The acid solutions presented higher $\mathrm{Cu}$ extractive capacity for all tested substrates. Among the substrates, only the coir presented statistically significant values of $\mathrm{R}^{2}$ for all the extraction methods. However, when the substrates were analyzed together, all methods presented statistically significant regression coefficients $\left(\mathrm{R}^{2}\right)$ higher than 0.51 , indicating that all the extraction methods can be used to quantify available $\mathrm{Cu}$ on these substrates.
\end{abstract}

Keywords: extracting agents, chemical analysis, micronutrient, coir, pinus bark, and peat.

\section{(Recebido para publicação em 22 de agosto de 2007; aceito em 23 de abril de 2008)}

$\mathrm{N}$ as últimas décadas, a produção vegetal expandiu-se com o desenvolvimento da automação e modernização dos sistemas de cultivo em ambiente protegido, passando a trabalhar em escalas mais amplas para atender um mercado que vem crescendo significativamente como a produção de mudas florestais, frutíferas e hortícolas. A produção dessas mudas transformou-se em agronegócio rentável no Brasil e a indústria foi compelida a desenvolver produtos alinhados com as exigências das novas demandas.

No passado, ao preencher um recipiente com solo mineral, o produtor buscava um material que permitia fixação e que fornecesse nutrientes para as plantas. Entretanto, devido a problemas relacionados à presença de ervas daninhas, pragas e doenças, o solo vem sendo substituído por outros materiais que têm por finalidade fixar as plantas e oferecer condições físicas adequadas ao seu desenvolvimento. Em cultivo de plantas em ambiente protegido é comum a utilização de substratos. O termo substrato aplica-se a todo material sólido, distinto do solo, podendo ser natural, sintético (espuma fenólica, lã de rocha), residual (esterco, bagaço de cana, fibras de algodão), mineral (perlita e vermiculita) ou orgânico (turfa, casca de árvores decompostas, fibra de coco). Esse material colocado em um recipiente em forma pura ou em mistura permite o desenvolvimento do sistema radicular, desempenhando, portanto, um papel de suporte às plantas (Abad \& Nogueira, 1998). Como características desejáveis, os substratos devem apresentar baixo custo, estar próximo às regiões de consumo, ter boa capacidade de troca de cátions, adequada aeração e retenção de água e isento de ervas daninhas (Konduru et al., 1999; Booman, 2000; Gonçalves et al., 2000).

No mercado brasileiro existe uma diversidade de substratos, principalmente no que se refere às características químicas e físicas e a sua origem, fazendo com que esses materiais sejam bastante complexos em termos de manejo de adubação. Dentre os problemas nutricionais, a falta ou o excesso de cobre $(\mathrm{Cu})$ é um dos que mais tem afetado a produção de mudas, principalmente de 
hortaliças e de citros. Boaventura (2003), estudando a demanda por nutrientes de mudas cítricas produzidas em substratos, verificou que aos 250 dias após o transplante, o porta-enxerto limão cravo que recebeu fertirrigação havia acumulado $20 \%$ mais $\mathrm{Cu}$ em relação ao que recebeu fertilizante de liberação lenta. Para o porta-enxerto citrumelo "Swingle" o acúmulo de $\mathrm{Cu}$ via fertirrigação foi $37 \%$ superior quando comparado ao mesmo porta-enxerto sob fertilizante de liberação lenta. Maiorano (2003), fazendo comparações entre o substrato versus inoculação com micorrizas, concluiu que as plantas de limão Cravo não micorrizadas cultivadas no substrato à base de fibra de coco 47, apresentaram os maiores acúmulos de $\mathrm{Cu}$ na parte aérea.

Para avaliar a disponibilidade de $\mathrm{Cu}$ em substratos, vários métodos têm sido testados. O procedimento que utiliza água, nas suas várias relações substrato:água, não tem sido muito eficiente (Abreu et al., 2007a). Neste procedimento de extração, geralmente os valores de Cu extraídos são baixos, próximos ao limite de detecção do aparelho, e as correlações entre os teores de $\mathrm{Cu}$ extraídos do substrato com os da planta são baixas.

Recentemente, iniciaram as pesquisas usando reagentes comumente empregados em extrair o $\mathrm{Cu}$ da fração orgânica dos solos, como o DTPA. Este é um aspecto importante porque a grande maioria dos substratos comerciais é de origem orgânica. Embora, o DTPA, método testado por Alt \& Peters (1993), seja empregado por vários países da Comunidade Européia para determinação dos micronutrientes em substratos, os resultados com substratos brasileiros não são muito consistentes (Abreu et al., 2007b). Para os substratos fibra de coco, casca de pinus e turfa nâo houve correlação significativa entre o teor de $\mathrm{Cu}$ extraído pelo DTPA e o teor deste na parte aérea da rúcula (Abreu et al., 2007b). Portanto, torna-se necessário buscar métodos alternativos que possam ser eficientes em avaliar a disponibilidade de $\mathrm{Cu}$ em substratos. Nesse contexto, a água oxigenada $\left(\mathrm{H}_{2} \mathrm{O}_{2}\right)$ a $30 \%+$ ácido nítrico $\left(\mathrm{HNO}_{3}\right)$ (Tessier et al., 1979), o pirofosfato de potássio
$\left(\mathrm{K}_{4} \mathrm{P}_{2} \mathrm{O}_{7}\right)($ Bascomb, 1968) e o hipoclorito de sódio $(\mathrm{NaOCl})$ a 5,8\% pH 8,5 + DTPA 0,025 mol L-1 (Shuman, 1983), comumente usados para determinar o $\mathrm{Cu}$ ligado à matéria orgânica do solo, poderão ser metodologias promissoras. $\mathrm{O}$ objetivo da presente pesquisa foi avaliar a disponibilidade de Cu em substratos, usando diferentes extratores e a planta teste rúcula; e avaliar a eficiência dos extratores utilizados para quantificar o $\mathrm{Cu}$ ligado à matéria orgânica de solos, em esquemas de fracionamento, em determinar o Cu disponível em substratos.

\section{MATERIAL E MÉTODOS}

O experimento foi realizado em casa de vegetação do Instituto Agronômico, Campinas-SP, e como substratos foram utilizadas a turfa, casca de pinus, e a fibra de coco, sendo todos os materiais indicados para hortaliças. A acidez da turfa e da fibra de coco foi corrigida na própria fábrica e apresentava $\mathrm{pH}$ em água de 5,2 e 5,6 respectivamente, entretanto a casca de pinus que apresentou o pH em água de 3,8 recebeu $180 \mathrm{~g}$ de calcário dolomítico para cada $18 \mathrm{~L}$ de substrato elevando o $\mathrm{pH}$ para 5,7.

Em cada material determinou-se a densidade úmida (aquela dos substratos que estavam nos vasos) e a densidade seca a $65^{\circ} \mathrm{C}$ para fins analíticos.

$\mathrm{O}$ delineamento experimental foi inteiramente ao acaso, em esquema fatorial 3 X 5, com 4 repetições. Os tratamentos constituíram de três substratos (turfa, casca de pinus e fibra de coco) e cinco doses de $\mathrm{Cu}$ : 0; 0,5; 1,0; 2,0 e 3,0 $\mathrm{mg}$ de $\mathrm{Cu}$ por $\mathrm{dm}^{3}$ de substrato, usando como fonte o sulfato de cobre. Os substratos permaneceram incubados por 40 dias mantendo-se o teor de umidade próxima à capacidade de recipiente, por meio de pesagem diária. $\mathrm{Na}$ definição do teor de água a aplicar em cada vaso (capacidade de recipiente) optou-se pelo método prático, ou seja, as amostras de substrato foram misturadas com água deionizada e, apertadas levemente nas mãos, até que a água escorresse por entre os dedos. A quantidade de água gasta para chegar a este ponto foi considerado como capacidade de recipiente.

Decorrido esse período, fez-se à adubação básica contendo macro e micro- nutrientes, com exceção do $\mathrm{Cu}$. As seguintes doses, em $\mathrm{mg} \mathrm{dm}^{-3}$, foram aplicadas: 28,6 de $\mathrm{N}$ e 80 de $\mathrm{K}\left(\mathrm{KNO}_{3}\right) ; 250$ de $\mathrm{P}$ (superfosfato simples); 381 de $\mathrm{S}$ (de todas as fontes que continham sulfato); 2,0 de $\mathrm{Zn}\left(\mathrm{ZnSO}_{4}\right) ; 0,4$ de B $\left(\mathrm{H}_{3} \mathrm{BO}_{3}\right) ; 2,0$ de $\mathrm{Mn}\left(\mathrm{MnCl}_{2}\right) ; 1,0$ de $\mathrm{Fe}$ $\left.\left[\mathrm{Fe}\left(\mathrm{NH}_{4}\right)_{2}\left(\mathrm{SO}_{4}\right)_{2} \cdot 6 \mathrm{H}_{2} \mathrm{O}\right)\right]$. Após, o material permaneceu incubado por uma semana de maneira semelhante ao realizado para a adubação com $\mathrm{Cu}$.

Como planta teste utilizou-se a rúcula (Eruca sativa L.), cultivada, em vasos com capacidade de $3 \mathrm{~L}$.

Durante todo o ensaio, em intervalos de 4 dias, aplicou-se uma solução nutritiva de acordo com (Furlani et al., 1999). As seguintes doses, em $\mathrm{mg} \mathrm{dm}^{-3}$, foram aplicadas: 68,8 de $\mathrm{N} ; 18,7 \mathrm{de} \mathrm{P}$; 99,1 de $\mathrm{K} ; 145,13$ de $\mathrm{Ca} ; 69,17$ de $\mathrm{Mg}$; 92,7 de $\mathrm{S} ; 1,41$ de Fe; 0,06 de Zn; 0,24 de Mn; 0,19 de B e 0,03 de Mo.

Sessenta dias após o plantio realizouse a colheita da parte aérea, e as amostras foram lavadas com água + detergente, água corrente e água e, em seguida, pesadas e trituradas em moinho tipo Wiley e submetida à digestão via seca conforme (Abreu et al., 1997). Nos extratos, foram feitas determinações dos elementos por espectrometria de emissão por plasma ICP-OES, exceto o $\mathrm{K}$ que foi por fotometria de emissão por chama.

Para extrair o $\mathrm{Cu}$ dos substratos utilizaram-se os seguintes procedimentos:

DTPA/ $\mathrm{CaCl}_{2}$ pH 2,6 (CAT) - de acordo com Alt \& Peters (1993). Em 60 $\mathrm{cm}^{3}$ de substrato (volume determinado em massa de acordo com a densidade calculada) adicionaram-se $300 \mathrm{~mL}$ de solução extratora de DTPA 0,002 mol $\mathrm{L}^{-1} \mathrm{CaCl}_{2}$ 0,01 mol L-1 $\mathrm{pH} 2,6$. A suspensão foi agitada por uma hora em agitador horizontal de 220 rpm e, logo após, filtrada.

DTPA/TEA/CaCl 7,3 - método utilizado para determinação de micronutrientes no solo (Lindsay \& Norvell, 1978). Em $50 \mathrm{~cm}^{3}$ de substrato (volume determinado em massa de acordo com a densidade calculada) adicionaram-se $100 \mathrm{~mL}$ da solução (DTPA 0,005 mol L${ }^{1}+$ trietanolamina $0,1 \mathrm{~mol} \mathrm{~L}{ }^{-1}+\mathrm{CaCl}_{2}$ $\left.0,01 \mathrm{~mol} \mathrm{~L}^{-1}, \mathrm{pH} 7,3\right)$. Essa mistura foi agitada por duas horas em agitador horizontal a $220 \mathrm{rpm}$ seguindo-se de filtragem. 
Tabela 1. Teor de $\mathrm{Cu}$ extraído do substrato $\left(\mathrm{mg} \mathrm{kg}^{-1}\right)$ pelos diferentes métodos, em função das doses de cobre aplicadas nos substratos turfa, casca de pinus e fibra de coco. Campinas, IAC, 2006.

\begin{tabular}{|c|c|c|c|c|c|c|}
\hline $\begin{array}{l}\text { Dose de } \\
\text { Cu mg dm } \\
\text {.3 }\end{array}$ & DTPA 2,6 & DTPA 7,3 & $\mathrm{K}_{4} \mathbf{P}_{2} \mathbf{O}_{7}$ & $\begin{array}{c}\mathrm{HNO}_{3}+ \\
\mathrm{H}_{2} \mathrm{O}_{2}\end{array}$ & $\begin{array}{c}\mathrm{NaOCl+} \\
\text { DTPA }\end{array}$ & $\begin{array}{c}\mathrm{HNO}_{3}+ \\
\mathrm{HClO}_{4}\end{array}$ \\
\hline \multicolumn{7}{|c|}{ Turfa, mg kg-1 } \\
\hline 0 & 0,61 & 0,51 & 0,06 & 6,66 & 4,10 & 5,48 \\
\hline 0,5 & 0,68 & 0,66 & 0,06 & 8,86 & 4,80 & 7,77 \\
\hline 1,0 & 0,78 & 0,70 & 0,54 & 10,87 & 5,70 & 8,52 \\
\hline 2,0 & 0,77 & 0,88 & 0,03 & 14,90 & 8,10 & 11,59 \\
\hline 3,0 & 0,95 & 1,36 & 1,03 & 18,83 & 9,70 & 14,57 \\
\hline Média & 0,76 & 0,82 & 0,34 & 12,02 & 6,48 & 9,59 \\
\hline \multicolumn{7}{|c|}{ Casca de Pinus, $\mathrm{mg} \mathrm{kg}^{-1}$} \\
\hline 0 & 0,47 & 0,43 & 2,48 & 11,93 & 8,27 & 10,38 \\
\hline 0,5 & 0,69 & 0,59 & 3,25 & 16,25 & 9,75 & 11,72 \\
\hline 1,0 & 0,83 & 0,71 & 2,88 & 18,25 & 12,85 & 14,60 \\
\hline 2,0 & 1,22 & 1,09 & 3,00 & 19,65 & 17,25 & 19,08 \\
\hline 3,0 & 1,58 & 1,40 & 3,22 & 21,02 & 21,80 & 23,03 \\
\hline Média & 0,96 & 0,84 & 2,97 & 17,42 & 13,98 & 15,76 \\
\hline \multicolumn{7}{|c|}{ Fibra de Coco, $\mathrm{mg} \mathrm{kg}^{-1}$} \\
\hline 0 & 1,31 & 1,77 & 4,60 & 27,35 & 20,35 & 17,97 \\
\hline 0,5 & 1,66 & 2,29 & 4,83 & 34,30 & 25,10 & 19,44 \\
\hline 1,0 & 2,09 & 2,87 & 6,90 & 44,65 & 34,00 & 27,03 \\
\hline 2,0 & 2,87 & 4,13 & 7,13 & 63,35 & 43,85 & 30,89 \\
\hline 3,0 & 3,88 & 5,28 & 9,45 & 76,00 & 58,60 & 39,87 \\
\hline Média & 2,36 & 3,27 & 6,58 & 49,13 & 36,38 & 27,04 \\
\hline
\end{tabular}

Pirofosfato de potássio - $\mathbf{K}_{4} \mathbf{P}_{2} \mathbf{O}_{7}$, procedimento adaptado por Bascomb (1968) para determinação de $\mathrm{Cu}$ ligado à matéria orgânica do solo em esquema de fracionamento. Em um grama de amostra seca e moída adicionaram-se $100 \mathrm{~mL}$ de $\mathrm{K}_{4} \mathrm{P}_{2} \mathrm{O}_{7} 0,1 \mathrm{~mol} \mathrm{~L}^{-1}$. Os frascos contendo a mistura foram postos para agitar, por 16 horas, em agitador horizontal de $200 \mathrm{rpm}$. Para obtenção de um filtrado límpido, o pH do extrato foi corrigido para 1,5 usando o ácido clorídrico concentrado, depois de 24 horas de repouso (para decantação) a solução foi filtrada.

Ácido nítrico e água oxigenada $\mathrm{HNO}_{3} / \mathrm{H}_{2} \mathrm{O}_{2}-$ método adaptado de Tessier et al. (1979) para determinação de $\mathrm{Cu}$ ligado à matéria orgânica do solo, em esquemas de fracionamentos. Em um grama de amostra seca adicionaramse $3 \mathrm{~mL}$ de $\mathrm{HNO}_{3} 0,02 \mathrm{~mol} \mathrm{~L}^{-1}+5 \mathrm{~mL}$ de $\mathrm{H}_{2} \mathrm{O}_{2}$, a pH 2,0, sendo aquecidas a $85^{\circ} \mathrm{C} \pm 2^{\circ} \mathrm{C}$ por 2 horas, agitando-se ocasionalmente. O processo foi repetido por 3 vezes até a completa queima da matéria orgânica. Depois, filtrou-se o extrato e o completou-se o volume para 20 $\mathrm{mL}$, usando-se água deionizada. de $\mathrm{Cu}$ foi determinada usando a espectrometria de emissão por plasma (ICP-OES).

Os teores extraídos pelos métodos DTPA 2,6 e DTPA 7,3, expressos em volume, foram transformados para massa tendo-se como finalidade a comparação dos resultados entre métodos.

$\mathrm{Na}$ análise de variância empregouse o software SAS v.6.11 (SAS, 1996). A análise de regressão para as variáveis, teor de $\mathrm{Cu}$ extraído dos substratos pelos diferentes métodos e o seu teor na planta, foi realizada considerando o substrato individualmente ou o conjunto (os três materiais). Nas análises de regressão linear ou polinomial, escolheu-se o modelo de maior significância $(* \mathrm{p}<0,05 \mathrm{ou}$ $* * \mathrm{p}<0,01)$.

\section{RESULTADOS E DISCUSSÃO}

Houve grande variabilidade nos teores de $\mathrm{Cu}$ extraídos pelos diferentes métodos empregados, independentemente do substrato (Tabela 1). Observou-se a seguinte ordem decrescente de extração: $\mathrm{HNO}_{3}+\mathrm{H}_{2} \mathrm{O}_{2}>\mathrm{HNO}_{3}+$ $\mathrm{HClO}_{4}>\mathrm{NaOCl}+$ DTPA $>$ DTPA 7,3 > DTPA 2,6 $>\mathrm{K}_{4} \mathrm{P}_{2} \mathrm{O}_{7}$ para a turfa; $\mathrm{HNO}_{3}$ $+\mathrm{H}_{2} \mathrm{O}_{2}>\mathrm{HNO}_{3}+\mathrm{HClO}_{4}>\mathrm{NaOCl}+$ DTPA $>\mathrm{K}_{4} \mathrm{P}_{2} \mathrm{O}_{7}>$ DTPA 2,6 > DTPA 7,3 para a casca de pinus; $\mathrm{HNO}_{3}+\mathrm{H}_{2} \mathrm{O}_{2}$ $>\mathrm{NaOCl}+\mathrm{DTPA}>\mathrm{HNO}_{3}+\mathrm{HClO}_{4}>$ $\mathrm{K}_{4} \mathrm{P}_{2} \mathrm{O}_{7}>$ DTPA 7,3 > DTPA 2,6 para a fibra de coco. Essa sequiência de extração era esperada, uma vez que os ácidos nítrico e perclórico são agentes oxidantes muito fortes, oxidam a matéria orgânica liberando todo o $\mathrm{Cu}$ ligado aos compostos orgânicos.

Quanto ao agente quelante, DTPA, ele deve ter extraído somente o $\mathrm{Cu}$ fracamente ligado à matéria orgânica. Torna-se importante mencionar que o $\mathrm{Cu}$ complexado à matéria orgânica apresenta diferentes constantes de estabilidade (Hao et al., 1980; Haddad \& Evans, 1993). A constante $\left(K_{t}\right)$ é definida como a energia liberada durante a ligação $\mathrm{Cu}$ ligante. Os ácidos húmicos de diferentes fontes variam quanto ao número de grupos funcionais e a sua massa molecular, fatores esses que alteram a natureza e a estabilidade do complexo formado, bem como a extração e disponibilidade de $\mathrm{Cu}$ para as plantas. $\mathrm{Na}$ 
Tabela 2. Equações de regressão para o Cu extraído dos substratos pelos diferentes métodos em função do Cu extraído pela planta (concentração e acúmulo) onde: y é o $\mathrm{Cu}$ na planta $\left(\mathrm{mg} \mathrm{kg}^{-1}\right)$, e x é a dose de $\mathrm{Cu}$ extraído do substrato em $\left(\mathrm{mg} \mathrm{kg}^{-1}\right)$ com exceção do DTPA 2,6 e 7,3 que apresentaram valores expressos em volume $\left(\mathrm{mg} \mathrm{dm}^{-3}\right)$ e massa $\left(\mathrm{mg} \mathrm{kg}^{-1}\right)$. Campinas, IAC, 2006.

\begin{tabular}{|c|c|c|c|c|}
\hline Métodos & Equação Cu-planta (concentração) & $\mathbf{R}^{\mathbf{2}}$ & Equação Cu-planta (acumulado) & $\mathbf{R}^{\mathbf{2}}$ \\
\hline \multicolumn{5}{|c|}{ Turfa } \\
\hline DTPA 2,6 v/v & $y=73,31 x 2-126,23 x+58,67$ & $0,29^{\text {ns }}$ & $y=121,66 x 2-207,61 x+108,04$ & $0,22^{\text {ns }}$ \\
\hline DTPA 2,6 & $y=87,62 x 2-145,7 x+64,62$ & $0,43^{\text {ns }}$ & $y=132,4 x 2-218,26 x+109,17$ & $0,27^{\text {ns }}$ \\
\hline DTPA $7,3 \mathrm{v} / \mathrm{v}$ & $y=11,51 x 2-24,50 x+17,03$ & $0,55^{\mathrm{ns}}$ & $y=14,10 x 2-29,11 x+34,64$ & $0,22^{\text {ns }}$ \\
\hline DTPA 7,3 & $y=13,36 x 2-26,08 x+16,76$ & $0,56^{\mathrm{ns}}$ & $y=17,12 x 2-32,47 x+34,96$ & $0,25^{\text {ns }}$ \\
\hline $\mathrm{K}_{4} \mathrm{P}_{2} \mathrm{O}_{7}$ & $y=24,86 x 2-59,83 x+39,53$ & $0,53^{\text {ns }}$ & $y=28,49 \times 2-66,86 \times 58,38$ & $0,21^{\text {ns }}$ \\
\hline $\mathrm{HNO}_{3}+\mathrm{H}^{2} \mathrm{O}_{2}$ & $y=0,07 x 2-1,91 x+16,52$ & $0,69^{\text {ns }}$ & $y=0,10 x 2-2,43 x+34,67$ & $0,31^{\text {ns }}$ \\
\hline $\mathrm{NaOCl}+\mathrm{DTPA}$ & $y=0,31 x 2-4,42 x+19,56$ & $0,54^{\mathrm{ns}}$ & $y=0,36 x 2-4,86 x+36,02$ & $0,20^{\text {ns }}$ \\
\hline $\mathrm{HNO} 3+\mathrm{HClO} 4$ & $y=0,16 x 2-3,23 x+20,26$ & $0,55^{\mathrm{ns}}$ & $y=0,25 x 2-4,88 x+42,89$ & $0,51^{\mathrm{ns}}$ \\
\hline \multicolumn{5}{|c|}{ Casca de Pinus } \\
\hline DTPA 2,6 v/v & $y=-3,80 x 2+7,70 x+3,79$ & $0,19^{\text {ns }}$ & $y=-21,53 x 2+45,70 x+6,71$ & $0,38^{\text {ns }}$ \\
\hline DTPA 2,6 & $y=-3,54 x 2+7,32 x+3,86$ & $0,18^{\text {ns }}$ & $y=-19,80 x 2+43,06 x+7,37$ & $0,37^{\mathrm{ns}}$ \\
\hline DTPA $7,3 \mathrm{v} / \mathrm{v}$ & $y=-5,21 x 2+9,37 x+3,48$ & $0,17^{\mathrm{ns}}$ & $y=-37,01 x 2+68,14 x+1,23$ & $0,29^{\text {ns }}$ \\
\hline DTPA 7,3 & $y=-5,03 x 2+9,24 x+3,48$ & $0,16^{\mathrm{ns}}$ & $y=-28,28 x 2+54,31 x+5,06$ & $0,32^{\text {ns }}$ \\
\hline $\mathrm{K}_{4} \mathrm{P}_{2} \mathrm{O}_{7}$ & $y=-11,94 x 2+67,96 x-88,51$ & $0,51^{\mathrm{ns}}$ & $y=-54,57 x 2+315,39 x-423,86$ & $0,59^{\text {ns }}$ \\
\hline $\mathrm{HNO}_{3}+\mathrm{H}_{2} \mathrm{O}_{2}$ & $y=-0,03 x 2+1,21 x-3,26$ & $0,14^{\mathrm{ns}}$ & $y=-0,20 x 2+7,25 x-36,53$ & $0,38^{\text {ns }}$ \\
\hline $\mathrm{NaOCl}+\mathrm{DTPA}$ & $y=-0,03 x 2+0,96 x+0,58$ & $0,27^{\mathrm{ns}}$ & $y=-0,16 x 2+4,93 x-7,07$ & $0,43^{\text {ns }}$ \\
\hline $\mathrm{HNO}_{3}+\mathrm{HClO}_{4}$ & $y=-0,04 x 2+1,28 x-2,86$ & $0,29^{\text {ns }}$ & $y=-0,19 x 2+6,48 x-24,08$ & $0,45^{\text {ns }}$ \\
\hline \multicolumn{5}{|c|}{ Fibra de Coco } \\
\hline DTPA 2,6 v/v & $y=3,09 x+10,22$ & $0,95^{\star *}$ & $y=13,06 x+52,78$ & $0,89 * *$ \\
\hline DTPA 2,6 & $y=1,83 x+10,49$ & $0,95^{* *}$ & $y=8,49 x+52,38$ & $0,91^{*}$ * \\
\hline DTPA $7,3 \mathrm{v} / \mathrm{v}$ & $y=2,04 x+10,54$ & $0,94^{* *}$ & $y=8,62 x+54,21$ & $0,87^{* *}$ \\
\hline DTPA 7,3 & $y=1,30 x+10,58$ & $0,95^{* *}$ & $y=6,10 x+52,48$ & 0,90 ** \\
\hline $\mathrm{K}_{4} \mathrm{P}_{2} \mathrm{O}_{7}$ & $y=0,93 x+8,71$ & $0,90^{* *}$ & $y=4,06 x+45,52$ & $0,90 * *$ \\
\hline $\mathrm{HNO}_{3}+\mathrm{H}_{2} \mathrm{O}_{2}$ & $y=0,09 x+10,31$ & $0,92^{* *}$ & $y=0,43 x+51,28$ & 0,90 ** \\
\hline $\mathrm{NaOCl}+\mathrm{DTPA}$ & $y=0,12 x+10,34$ & $0,95^{* *}$ & $y=0,52 x+53,22$ & 0,90 ** \\
\hline $\mathrm{HNO}_{3}+\mathrm{HClO}_{4}$ & $y=0,21 x+9,19$ & $0,93^{* *}$ & $y=0,90 x+47,95$ & $0,90^{* *}$ \\
\hline
\end{tabular}

** significativo a $1 \%$ pelo teste $\mathrm{t}$

Tabela 1 observa-se que o DTPA pH 7,3 extraiu mais $\mathrm{Cu}$ que o DTPA pH 2,6 quando os substratos eram a turfa e a fibra de coco. Tais resultados podem ser justificados pela alta estabilidade do complexo DTPA-Cu, formado acima de pH 7,0 (Tisdale et al., 1985). De acordo com Norvell (1991), em solos a estabilidade do quelante DTPA com o Cu é praticamente nula em meio ácido, devido à competição com o Fe, justificando a menor extração de $\mathrm{Cu}$ pelo método DTPA pH 2,6.

Haynes \& Swift (1985), comparando a capacidade dos métodos $\mathrm{CaCl}_{2} 0,05$ mol L-1, HCl 0,1 mol L-1, DTPA 0,005 mol L ${ }^{-1}$ e EDTA $0,04 \mathrm{~mol} \mathrm{~L}^{-1}$ em extrair nutrientes do substrato turfa cultivada com mirtilo azul (Vaccinium corymbosum L cv. Blueray), verificaram que o DTPA $0,005 \mathrm{~mol} \mathrm{~L}^{-1}$ teve comportamento semelhante ao $\mathrm{HCl} 0,1 \mathrm{~mol}$ $\mathrm{L}^{-1}$. Já o EDTA $0,04 \mathrm{~mol} \mathrm{~L}^{-1}$ apresentou valores de extração superiores ao $\mathrm{CaCl}_{2}$ $0,05 \mathrm{~mol} \mathrm{~L}^{-1}$. Alt \& Peters (1993) compararam a capacidade extrativa de $\mathrm{Cu}$ pelo método DTPA pH 2,6 em relação aos métodos $\mathrm{CaCl}_{2}$, DTPA pH 7,3 + $\mathrm{CaCl}_{2}$ e $\mathrm{NH}_{4} \mathrm{OAc}$, usando como teste um substrato composto por turfa e argila. Eles observaram que o DTPA pH 2,6 apresentou maior capacidade de extração de $\mathrm{Cu}$ que o $\mathrm{NH}_{4} \mathrm{OAc}$, sendo a extração desse semelhante ao DTPA pH 7,3. Contudo, Narwal \& Singh (1998) observaram uma relação inversa entre a adição de matéria orgânica (turfa) aos solos e o teor de Cu extraído pelo DTPA.

Outro fator que chama atenção, são os maiores valores de extração de $\mathrm{Cu}$ obtidos pelos métodos $\mathrm{NaOCl}+$ DTPA, $\mathrm{HNO}_{3}+\mathrm{H}_{2} \mathrm{O}_{2}$ e $\mathrm{HNO}_{3}+\mathrm{HClO}_{4}$, principalmente na fibra de coco (Tabela 1). Abreu et al. (2005), comparando a extração do $\mathrm{HNO}_{3}+\mathrm{HClO}_{4}$ com a do $\mathrm{HNO}_{3}$ (em sistema fechado com forno de microondas), usando substratos de origem orgânica e inorgânica, concluíram que o extrator $\mathrm{HNO}_{3}+\mathrm{HClO}_{4}$ extraiu 26\% a mais de $\mathrm{Cu}$ que o método $\mathrm{HNO}_{3}$. Contudo, os resultados obtidos neste trabalho para a fibra de coco podem ser justificados, em parte, pela densidade do substrato. A turfa, a casca de pinus e a fibra de coco apresentaram como valores de densidade seca 0,440 , 0,340 e $0,110 \mathrm{~kg} \mathrm{~m}^{-3}$, respectivamente. Quanto menor a densidade maior o volume de amostra utilizado, podendo resultar em maior extração de $\mathrm{Cu}$, pelos extratores $\mathrm{NaOCl}+$ DTPA, $\mathrm{HNO}_{3}+$ $\mathrm{H}_{2} \mathrm{O}_{2}$ e $\mathrm{HNO}_{3}+\mathrm{HClO}_{4}$, na fibra de coco.

Fazendo-se uma avaliação separadamente, por substrato, da capacidade de cada método químico em avaliar a disponibilidade de $\mathrm{Cu}$ para a rúcula, verificam-se correlações significativas entre o Cu-planta (acumulado ou concentração) e o Cu extraído do substrato so- 


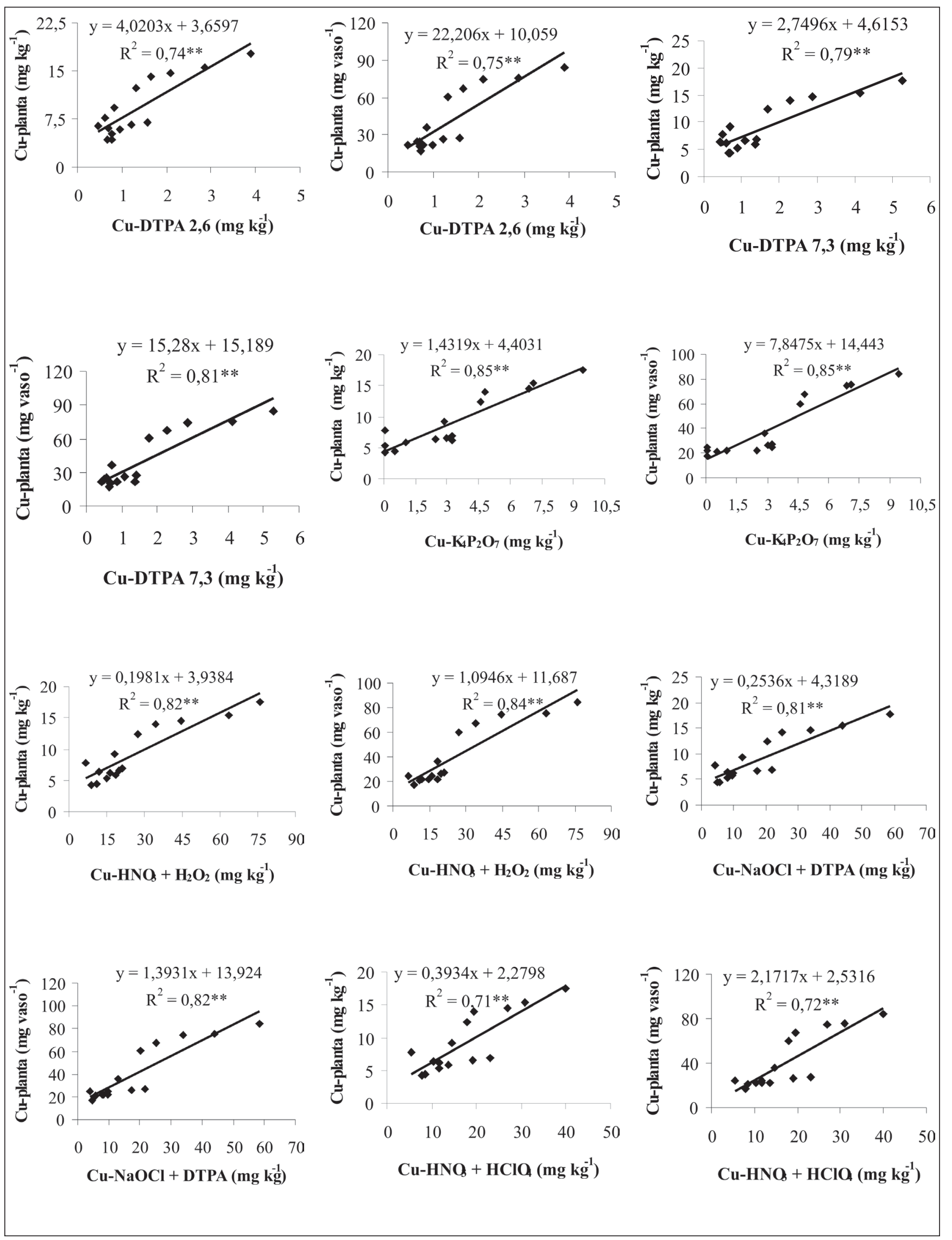

Figura 1. Relação entre a concentração ou acúmulo de $\mathrm{Cu}$ na parte aérea da rúcula e o Cu extraído dos substratos (turfa, casca de pinus e fibra de coco) usando diferentes métodos. Para todos os métodos de extração, os resultados de Cu-substrato foram expresso em mg $\mathrm{kg}^{-1}$. No caso do DTPA pH 2,6 e pH 7,3, os resultados também foram expressos em mg L-1. Campinas, IAC, 2006. 
mente para a fibra de coco (Tabela 2). Não foi possível explicar, por meio de modelos matemáticos, a variação encontrada na concentração de $\mathrm{Cu}$ na parte aérea da rúcula pelo uso da análise de $\mathrm{Cu}$ nos substratos casca de pinus e turfa, pelos métodos testados (Tabela 2). Resultados semelhantes foram encontrados por Abreu et al. (2007b), avaliando a capacidade do extrator DTPA 2,6 em determinar o $\mathrm{Cu}$ disponível para a rúcula desenvolvida nos substratos turfa e casca de pinus.

Quando os substratos foram analisados conjuntamente (Figura 1) todos os métodos apresentaram coeficientes de regressão significativos e maiores que 0,51 , sendo que os valores de $\mathrm{R}^{2}$ obtidos para o $\mathrm{Cu}$ acumulado na planta foi ligeiramente superiores aos valores obtidos para a concentração de Cu na planta. A eficiência dos extratores, avaliada pelos valores de correlação entre $\mathrm{Cu}$ planta e Cu-substrato, obedeceu a seguinte ordem decrescente: $\mathrm{K}_{4} \mathrm{P}_{2} \mathrm{O}_{7}>$ $\mathrm{HNO}_{3}+\mathrm{H}_{2} \mathrm{O}_{2}>\mathrm{NaOCl}+\mathrm{DTPA}>$ DTPA 7,3 > DTPA 2,6 $>\mathrm{HNO}_{3}+\mathrm{HClO}_{4}$ $>$ DTPA 7,3 (v/v) > DTPA 2,6 (v/v). Dentre os métodos testados, aqueles comumente utilizados para determinar $\mathrm{Cu}$ da fração orgânica do solo, em esquemas de fracionamento de metais do solo, foram os que apresentaram maiores valores de $\mathrm{R}^{2}$, destacando-se o $\mathrm{K}_{4} \mathrm{P}_{2} \mathrm{O}_{7}$ que melhor se relacionou com a concentração de $\mathrm{Cu}$ na planta e o seu acúmulo (Figura 1). Na seqüência, destacaram-se o $\mathrm{HNO}_{3}+\mathrm{H}_{2} \mathrm{O}_{2}$ e o $\mathrm{NaOCl}$ + DTPA com valores de coeficiente de determinação de $\mathrm{R}^{2}=0,82 * *$ e $0,81 * *$ respectivamente, para a relação entre a concentração de $\mathrm{Cu}$ na planta e o $\mathrm{Cu}$ no substrato (Figura 1).

Os valores de $\mathrm{R}^{2}$ foram mais baixos para os extratores cujos resultados foram expressos em volume (DTPA 2,6 v/v e DTPA 7,3 v/v) se comparados com os valores do DTPA 2,6 e DTPA 7,3, expressos em massa (Figura 1). Contudo, os pontos obtidos no gráfico entre o $\mathrm{Cu}$-planta em função do $\mathrm{Cu}$-substrato foram bem mais distribuídos nos métodos DTPA 2,6 v/v e DTPA 7,3 v/v, diferenciando dos demais métodos testados. Este fato mostra a importância da padronização do uso de volume ou massa tanto na pesagem da amostra para aná-
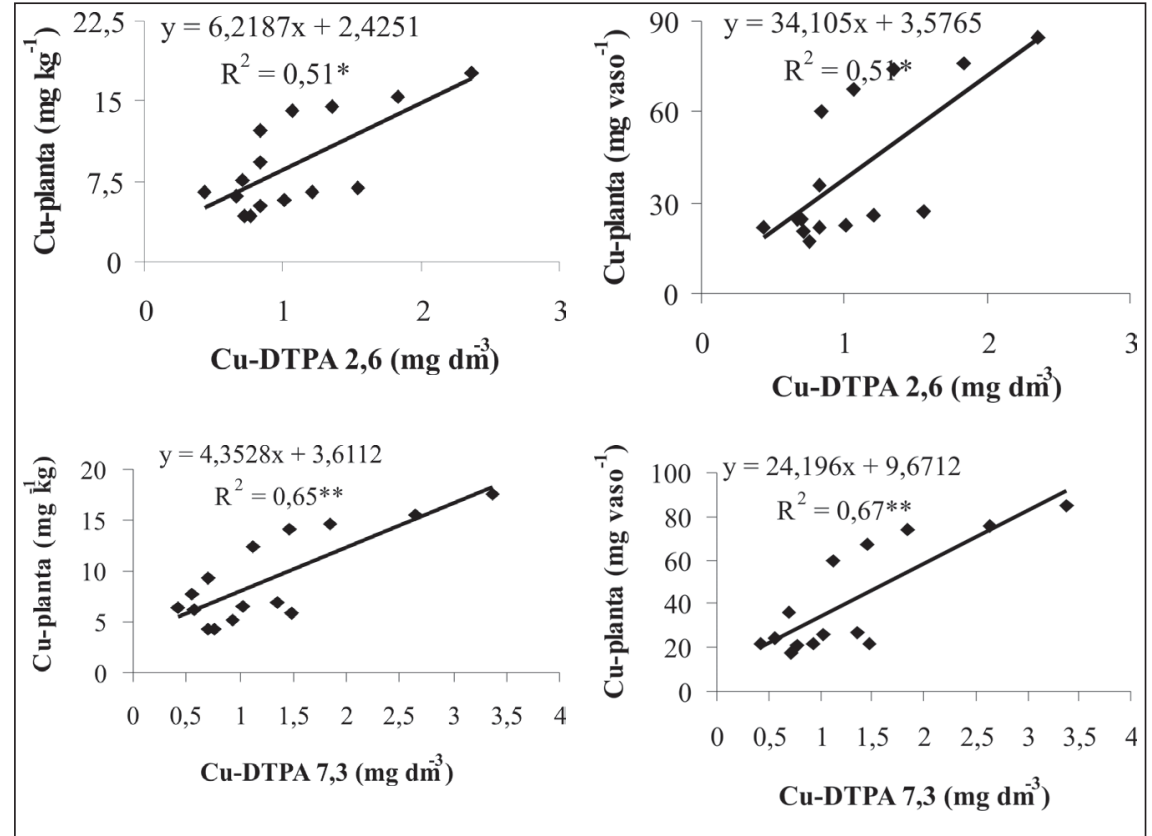

Figura 1. Continuação

lise como na expressão final dos resultados, principalmente para os substratos que geralmente têm densidade bem menor de 1,0 .

Além da eficiência do extrator em avaliar a disponibilidade do $\mathrm{Cu}$, medida pela relação entre o Cu extraído pela planta e $\mathrm{Cu}$ extraído pelo método químico, outros fatores são importantes quando se almeja selecionar métodos para uso em condições de rotina do laboratório. Esses aspectos são o tamanho da amostra empregada, a reprodutibilidade e repetibilidade do procedimento no laboratório, amplitude de variação, o tempo gasto na extração, o requerimento de equipamento especial, dentre outros.

Quanto à quantidade de amostra, os métodos $\mathrm{K}_{4} \mathrm{P}_{2} \mathrm{O}_{7}, \mathrm{NaOCl}+\mathrm{DTPA}$, $\mathrm{HNO}_{3}+\mathrm{H}_{2} \mathrm{O}_{2}$ e $\mathrm{HNO}_{3}+\mathrm{HClO}_{4}$ empregaram as menores massas, o que prejudica o procedimento analítico em condições de rotina laboratorial. Tal fato é relevante porque grande parte dos substratos é composta de vários materiais com características químicas e físicas diferenciadas (tamanho do material), o que interfere na homogeneidade de uma amostra pequena e conseqüentemente no teor extraído.

Por meio do coeficiente de variação tem-se uma idéia da precisão dos métodos. Os coeficientes de variação tive- ram uma amplitude de 3,49 a 23,19\% para a fibra de coco; 5,44 a 21,64\% para casca de pinus; e 9,26 a 179,08\% para a turfa. Além da própria influência do material, os altos coeficientes de variação observados na turfa, devem-se, em parte, aos baixos teores de Cu extraídos pelos diferentes métodos, levando a erro analíticos. Em geral, o método que obteve o maior valor de $\mathrm{CV}$ foi o $\mathrm{K}_{4} \mathrm{P}_{2} \mathrm{O}_{7}$, usando a turfa. A menor precisão do $\mathrm{K}_{4} \mathrm{P}_{2} \mathrm{O}_{7}$, pode estar relacionado às várias etapas analíticas para completar todo o procedimento; quanto maior os números de etapas no método maiores são as probabilidades de se cometer erros.

$\mathrm{O}$ extrator que apresentou maior amplitude de extração de $\mathrm{Cu}$ foi o $\mathrm{HNO}_{3}$ $+\mathrm{H}_{2} \mathrm{O}_{2}$ com uma variação de $6,66 \mathrm{mg}$ a $76,00 \mathrm{mg}$ (Figura 1). Em seguida, encontram-se os extratores $\mathrm{NaOCl}$ +DTPA, $\mathrm{HNO}_{3}+\mathrm{HClO}_{4}$, com variações de 4,10 mg a 58,60 mg e 5,48 mg a 39,87 mg respectivamente, derrubando a hipótese sobre a maior capacidade extrativa pelo método $\mathrm{HNO}_{3}+\mathrm{HClO}_{4}$. A menor amplitude de extração de $\mathrm{Cu}$ foi observada para o método DTPA 2,6 que apresentou uma amplitude de 0,61 a $3,88 \mathrm{mg}$ de $\mathrm{Cu}$.

Quanto ao tempo de extração, de maneira geral os métodos mais trabalhosos foram $\mathrm{K}_{4} \mathrm{P}_{2} \mathrm{O}_{7}, \mathrm{NaOCl}+\mathrm{DTPA}$, $\mathrm{HNO}_{3}+\mathrm{H}_{2} \mathrm{O}_{2}$. Para o $\mathrm{K}_{4} \mathrm{P}_{2} \mathrm{O}_{7}$, as amos- 
tras foram agitadas por 16 horas sem interrupção, o que dificulta a sua adoção em condições de rotina de um laboratório. Além disso, esse extrator apresentou sólidos em suspensão e foi necessário a adição de $\mathrm{HCl}$ concentrado para abaixar o $\mathrm{pH}$ das amostras para 1,5, deixando o método mais trabalhoso. $\mathrm{Na}$ utilização do $\mathrm{NaOCl}+$ DTPA, as amostras foram inicialmente colocadas em banho Maria, a $60^{\circ} \mathrm{C}$. Ao final do processo, o volume foi alterado, necessitando ajuste no volume final para não ocasionar erros analíticos na determinação final do $\mathrm{Cu}$, o que demandou tempo. $\mathrm{O}$ método que empregou o $\mathrm{HNO}_{3}+$ $\mathrm{H}_{2} \mathrm{O}_{2}$ foi laborioso (três etapas), sendo necessário acompanhamento constante na digestão, para que a amostra em alta temperatura, não vazasse para fora do tubo digestor. Entretanto, após todas esses critérios anteriormente citados sobre a escolha do melhor método o $\mathrm{HNO}_{3}+\mathrm{HClO}_{4}$ foi tido como o mais plausível para a determinação do $\mathrm{Cu}$ em substratos.

Com os resultados obtidos, podemos concluir que: a) Os extratores ácidos têm maior capacidade de extração de $\mathrm{Cu}$; b) Houve efeito do $\mathrm{pH}$ na extração de $\mathrm{Cu}$ pelos quelantes; c) $\mathrm{O}$ substrato fibra de coco foi o único material em que todos os extratores utilizados foram eficientes em avaliar a disponibilidade de $\mathrm{Cu}$ para a rúcula; d) A análise conjunta dos três substratos demonstrou a eficiência dos extratores utilizados em função dos teores (concentração e acúmulo) absorvidos pela planta; e) Considerando os vários aspectos importantes na seleção dos extratores para determinar o $\mathrm{Cu}$ em substratos, o melhor extrator foi o $\mathrm{HNO}_{3}$ $+\mathrm{HClO}_{4}$

\section{REFERÊNCIAS}

ABAD M; NOGUEIRA P. 1998. Substratos para el cultivo sin suelo y fertirrigacion. In: Cadahia, C. (Ed.) Fertirrigacion: Cultivos hortícolas y ornamentais. Madrid: Mundi-Prensa. p. 287342.

ABREU, M.F. 1997. Extração e determinação simultânea por emissão em plasma de nutrientes e elementos tóxicos em amostras de interesse agronômico. Campinas: Universidade Estadual de Campinas, 135p (Tese doutorado).

ABREU MF; FURLANI AC; ABREU CA; SANTOS PH; GONZÁLEZ AP. 2005. Total element concentration quantification in substrates. Acta Horticulturae 697: 315-319.

ABREU MF; ABREU CA; SARZI I; LINARES A. 2007 ${ }^{\text {. Extratores aquosos para a }}$ caracterização química de substratos para plantas. Horticultura Brasileira 25: 184-187.

ABREU MF; SANTOS PH; FURLANI PR; ABREU CA. 2007b. Extração de substratos para obtenção da concentração de micronutrientes disponíveis para a rúcula. Horticultura Brasileira 25: 411-417.

ALT D; PETERS I. 1993. Analysis of macro and trace elements in horticultural substrates by means of the $\mathrm{CaCl} / \mathrm{DTPA}$ (CAT) method. Acta Horticulturae 342: 287-292.

BASCOMB CL. 1968. Distributions of pyrophosphate-extractable Iron and organic carbon in soils of various groups. Journal of Soil Science 52: 19-26.

BOAVENTURA PSR. 2003. Demanda por nutrientes de mudas cítricas produzidas em substrato em ambiente protegido. Campinas: Instituto Agronômico. 62p (Tese mestrado).

BOOMAN JL. 2000. Evolução dos substratos usados em horticultura ornamental na Califórnia. In: KÄMPF NA; FERMINO MH. Substratos para plantas 1: A base da produção vegetal em recipientes, Porto Alegre. Anais. Porto Alegre: UFRGS, p. 46-65.

FURLANI PR; SILVEIRA LCP; BOLONHEZI D; FAQUIN V. 1999. Cultivo hidropônico de plantas. Campinas: Instituto Agronômico. 52p (Boletim Técnico, 180).

GONÇALVES JLM; SANTARELI EG; MORAES NETO SP; MANARA MP. 2000. Produção de mudas de espécies nativas: substratos, nutrição, sombreamento e fertilização. In: GONÇALVES JLM; BENEDETTI V. (Ed). Nutrição e fertilização florestal. Piracicaba: IPEF. p. 309-350.
HAO AV; BATES TE; SOON YK. 1980. Comparasion of extractants for plant available zinc, cadmium, nickel and copper in contamined soils. Soil Science Society of America Journal 44: 772-777.

HAYNES RJ; SWIFT RS. 1985. Effects of liming on the extratability of $\mathrm{Fe}, \mathrm{Mn}, \mathrm{Zn}$ and $\mathrm{Cu}$ from peat medium and growth and micronutrient uptake of highbush blueberry plants. Plant and Soil.

KONDURU S; EVANS MR; STAMPS RH. 1999. Coconut husk and processing effects on chemical and physical, properties of coconut coir dust. Horticulture Science 34: 88-90.

LINDSAY WL; NORVELL WA. 1978. Development of a DTPA soil test for zinc, iron, manganese, and copper. Soil Science Society of American Journal 42: 421-428.

MAIORANO JA. 2003. Utilização de substratos orgânicos comerciais na obtenção de mudas micorrizadas de limoeiro "Cravo" em ambiente protegido. Campinas: Instituto Agronômico. 63p (Tese mestrado)

NARWAL RP; SINGH BR. 1998. Effect organic materials on partitioning, extractability and plant uptake of metals in a alum shale soil. Water, Air, \& Soil Pollution 103: 405-421.

NORVELL WA. 1991. Reactions of metal chelates in soils and nutrient solutions. In: MORTVEDT JJ; COX FR; SHUMAN LM; WELCH RM. (Ed.) Micronutrientes in agriculture. Madison, Soil Science Society of America p. 187-227.

SAS INSTITUTE (Cary, United States). 1996. SAS/STAT user's guide: version 6.11. 4.ed. Cary, v.2. 842p.

SHUMAN LM. 1983. Sodium hypochlorite methods for extracting microelements associated with soil organic matter. Soil Science Society of American Journal 47: 656660.

TESSIER A; CAMPBELL PGC; BISSON M. 1979. Sequential extration procedure for the speciation of particulate trace metals. Analytical Chemistry 51: 844-851.

TISDALE SL; NELSON WL; BEATON JD. 1985. Soil Fertility and Fertilizers. New York: Macmillan, 754p. 